\title{
Simulação realística na capacitação de profissionais de enfermagem em sala de vacinação
}

\author{
Realistic simulation in the training of nursing professionals in a vaccination room \\ Simulación realista en la formación de profesionales de enfermería en una sala de vacunación
}

\author{
Samuel Barroso Rodrigues \\ ORCID: https://orcid.org/0000-0002-9832-5510 \\ Universidade Federal de São João del-Rei, Brasil \\ E-mail: samuelbarroso88@gmail.com \\ Gláucia Daniele Pereira Assis \\ ORCID: https://orcid.org/0000-0002-0582-2658 \\ Universidade Federal de São João del-ReiBrasil \\ E-mail: gpereiraassis@gmail.com \\ Brener Santos Silva \\ ORCID: https://orcid.org/0000-0003-4610-3227 \\ Universidade de São Paulo, Brasil \\ E-mail: brenersantos@usp.br \\ Gabriela Cunha Corrêa Freitas de Oliveira \\ ORCID: https://orcid.org/0000-0002-7009-4618 \\ Universidade Federal de São João del-ReiBrasil. \\ E-mail: gabyccunha@gmail.com \\ Laís Oliveira de Moraes Tavares \\ ORCID: https://orcid.org/0000-0002-6603-775X \\ Universidade Federal de São João del-Rei, Brasil \\ E-mail: laisoliveiramt@gmail.com \\ Gabriela Gonçalves Amaral \\ ORCID: https://orcid.org/0000-0002-9629-2815 \\ Universidade de São Paulo, Brasil \\ E-mail: g.amaral@usp.br \\ Valéria Conceição de Oliveira \\ ORCID: https://orcid.org/0000-0003-2606-9754 \\ Universidade Federal de São João del-Rei, Brasil \\ E-mail: valeriaoliveira@ufsj.edu.br \\ Eliete Albano de Azevedo Guimarães \\ ORCID: https://orcid.org/0000-0001-9236-8643 \\ Universidade Federal de São João del-Rei, Brasil \\ E-mail: elietealbano@ufsj.edu.br
}

\section{Resumo}

Este estudo buscou descrever a elaboração de um cenário de simulação realística para capacitação de profissionais de enfermagem em salas de vacinas. Trata-se de um estudo qualitativo realizado em salas de vacinação das unidades de atenção básica de um município localizado na Macrorregião Oeste de Minas Gerais, entre 2018 e 2019. Participaram da simulação seis enfermeiros e seis técnicos de enfermagem, além de cinco pesquisadores e cinco estudantes de pósgraduação. A simulação realística incluiu três etapas: 1) Pré-simulação; 2) Simulação e 3) Debriefing. Para a análise dos dados foi utilizada a Análise de Conteúdo. Os participantes da capacitação assumiram que a simulação realística é uma metodologia inovadora, participativa e muito próxima da realidade do cotidiano e representam uma melhor compreensão e reflexão quanto às técnicas e rotinas dos serviços de imunização. A simulação realística é uma metodologia de aprendizagem que aproxima os participantes da sua realidade. Esse fato pode facilitar maior apropriação do conhecimento, aprimorar as habilidades das práticas das salas de vacinação.

Palavras-chave: Enfermagem; Administração de serviços de saúde; Capacitação em serviço; Treinamento por simulação; Educação em enfermagem.

\footnotetext{
Abstract

This study sought to describe the development of a realistic simulation scenario for training nursing professionals in vaccination rooms. This is a qualitative study conducted in vaccination rooms of primary care units of a municipality located in the Western Macroregion of Minas Gerais, between 2018 and 2019. Six nurses and six nursing technicians, as well as five researchers and five graduate students participated in the simulation.. The realistic simulation included
} 
three stages: 1) Pre-simulation; 2) Simulation and 3) Debriefing. Content Analysis was used to analyze the data. The participants of the training assumed that the realistic simulation is an innovative methodology, participatory, and very close to the reality of everyday life, and represents a better understanding and reflection on the techniques and routines of immunization services. Realistic simulation is a learning methodology that brings participants closer to their reality. This fact can facilitate a greater appropriation of knowledge, improve the skills of the practices in vaccination rooms.

Keywords: Nursing; Health service administration; In-service training; Simulation training; Education nursing

\section{Resumen}

Este estudio tuvo como objetivo describir el desarrollo de un escenario de simulación realista para la formación de los profesionales de enfermería en las salas de vacunación. Se trata de un estudio cualitativo realizado en salas de vacunación de unidades de atención primaria de un municipio ubicado en la Macrorregión Oeste de Minas Gerais, entre 2018 y 2019. En la simulación participaron seis enfermeras y seis técnicos de enfermería, así como cinco investigadores y cinco estudiantes de posgrado. La simulación realista incluyó tres etapas: 1) Pre-simulación; 2) Simulación y 3) Debriefing. Se utilizó el análisis de contenido para analizar los datos. Los participantes en la formación asumieron que la simulación realista es una metodología innovadora, participativa y muy cercana a la realidad del día a día y que representa una mejor comprensión y reflexión respecto a las técnicas y rutinas de los servicios de vacunación. La simulación realista es una metodología de aprendizaje que acerca a los participantes a su realidad. Este hecho puede facilitar una mayor apropiación del conocimiento, mejorar las habilidades de las prácticas de las salas de vacunación.

Palabras clave: Enfermería; Administración de servicios de salud; Capacitación en servicios; Entrenamiento por simulación; Educación en enfermería.

\section{Introdução}

Ao longo da descentralização dos Sistemas de Informação em Saúde (SIS), estudos apontaram os registros eletrônicos como grandes potencializadores do planejamento e a tomada de decisões no cotidiano dos serviços (Pinheiro et al., 2016; Santos et al., 2017; Community Preventive Services Task Force, 2015). A implantação e difusão dessa inovação tecnológica deve ser estudada de tal modo que seja implementado um sistema informatizado eficiente e sustentável, capaz de se fazer presente em todo território nacional e auxiliar os gestores na construção de uma assistência à saúde de qualidade (Cavalcante et al., 2018).

Apesar das vantagens destes recursos, a literatura também sugere um distanciamento entre as expectativas e as reais melhorias que acontecem nestes serviços, sobretudo porque a compreensão dos profissionais sobre como tais instrumentos funcionam ainda é incipiente (Pinheiro et al., 2016; Sato, 2015). Por outro lado, embora haja intervenções voltadas à melhoria do manuseio dessas ferramentas, algumas têm sucesso e outras não, sendo fundamental compreender o porquê isso acontece (Santos et al., 2017).

Entre os SIS incluem-se aqueles capazes de registrar e gerenciar dados referentes à imunização. O Sistema de Informação de Imunização (SII) é um instrumento tecnológico gerencial que permite o registro individual do vacinado e de suas procedências; o resgate de não vacinados; o fornecimento de dados de coberturas vacinais e de Eventos Adversos PósVacinação (EAPV); a apuração e controle da validade de imunobiológicos utilizados na rede e o controle de estoque (Community Preventive Services Task Force, 2015; Sato, 2015; Braz, Domingues, Teixeira, \& Luna, 2016).

No Brasil, o registro de imunização é realizado por SIS próprio ou pelo Sistema de Informação do Programa Nacional de Imunizações (SIPNI). Tais sistemas informacionais ainda enfrentam desafios quanto à operacionalização, integralidade e qualidade dos dados (duplicidade e sub-registro), à garantia da confidencialidade das informações e aos padrões de interoperabilidade (Sato, 2015; Braz et al., 2016). Este fato pode estar relacionado aos profissionais que ainda não têm conhecimento suficiente para usar a tecnologia na sua totalidade, se restringindo apenas ao registro de dados (Community Preventive Services Task Force, 2015; Braz et al., 2016).

São por estas questões que devem ser buscadas outras estratégias de sensibilização dos profissionais de saúde para manuseio dos registros de informação. As estratégias de capacitação não convencionais, pautadas em modelos de ensino- 
aprendizagem integradores e participativos podem ser uma alternativa, uma vez que se desvinculam do modelo de ensino ou capacitações tradicionais, a exemplo das metodologias ativas (Souza, Silva, \& Silva, 2018). Estas são definidas como métodos pedagógicos de ensino-aprendizagem, nos quais o professor passa a ter papel de mediador e o estudante estabelece posição de sujeito principal neste processo (Alvarez, \& Moya, 2017).

Nesta perspectiva, a simulação realística se configura em uma metodologia ativa que auxilia a aproximação do profissional à sua realidade, e se configura em um método inovador de capacitação em serviço para profissionais de enfermagem (Jeffries, \& Rizzolo, 2006). Ademais, têm sido cada vez mais empregadas e se mostrado bastante adequadas para área da saúde, especialmente em situações cujo aprendizado depende da vivência prática para a compreensão dos temas abordados (Oliveira, Massaroli, Martini, \& Rodrigues, 2018; Cogo, Lopes, Perdomini, Flores, \& Santos, 2019).

Portanto, levando-se em consideração a necessidade da busca de estratégias de mudança nas práticas educativas (Cogo et al., 2019), a simulação realística pode ser uma intervenção efetiva para capacitar profissionais de saúde que estão envolvidos em salas de vacinação. A elaboração desta intervenção pode possibilitar não apenas a identificar as dificuldades de manuseio do SII, bem como incitar uma motivação maior no manuseio desta tecnologia, sobretudo pelo potencial sensibilizador da intervenção. Nesta perspectiva, o estudo objetivou descrever a elaboração de um cenário de simulação realística para capacitação de profissionais de enfermagem em salas de vacinação.

\section{Metodologia}

Trata-se de estudo descritivo com abordagem qualitativa (Pereira, Shitsuka, Parreira, \& Shitsuka, 2018) realizado nas salas de vacinação das unidades básicas de saúde (UBS) de um município localizado na Macrorregião Oeste de Minas Gerais, entre 2018 e 2019.

A simulação foi elaborada a partir do referencial teórico da Taxonomia de Bloom, proposta em três domínios principais: cognitivo, afetivo e psicomotor (Hanshaw \& Dickerson, 2020). O domínio cognitivo envolve a aquisição de novos saberes (aprendizado), sendo útil para resolução de problemas diários. Os objetivos deste domínio foram agrupados em categorias que possuem nível de hierarquização, mas que em determinadas situações podem assumir maior flexibilidade e a interpolação, a saber: 1) (re) lembrar; 2) entender; 3) aplicar; 4) analisar; 5) sintetizar; e 6) criar. O domínio afetivo envolve os sentimentos, ou seja, o emocional do aprendiz. Suas categorias são: receptividade, resposta, valorização, organização e caracterização. Segundo este domínio, somente por meio da aquisição de capacidades anteriores é possível avançar no processo de compreensão do evento. Por fim, o domínio psicomotor está relacionado ao domínio de manipulação, articulação e naturalização do fenômeno estudado, envolvendo basicamente as habilidades físicas (Hanshaw \& Dickerson, 2020). A simulação foi executada seguindo três etapas: 1) Pré-simulação; 2) Simulação e 3) Debriefing (Troncon \& Maffei, 2007).

\subsection{Pré-simulação}

Na pré-simulação procedeu-se a elaboração da simulação, seguida da execução da técnica com pesquisadores (teste piloto). Foi procedida à validação do cenário, tendo sido realizada a técnica Delphi (Scarparo et al., 2012) como um método de tomada de decisão em grupos de especialistas no tema a ser abordado, com o objetivo principal de chegar a um consenso acerca de determinado assunto. Desta forma, o cenário construído (descrito), o checklist do facilitador e o vídeo da simulação foram enviados a três enfermeiras especialistas (quantidade escolhida para se evitar situações de eventual empate na tomada de decisão), sendo duas expertises em imunização e uma em simulação, a fim de revisarem o conteúdo escrito e encenado, bem como a disposição dos participantes em cena. Estes especialistas foram escolhidos por meio da relevância de atuação e das publicações nas áreas supracitadas. As avaliações foram sustentadas pela Taxonomia de Bloom (Hanshaw \& Dickerson, 2020). 
Para procederem a avaliação, as especialistas receberam um formulário contendo campos para pontuarem acerca da clareza, objetividade, pertinência e organização do cenário descrito. Quanto ao vídeo, puderam considerar sobre as seguintes questões: itens necessários, porém ausentes; itens desnecessários e itens observados na encenação (ator, figurino e atuação). Posteriormente às avaliações, as observações e considerações das especialistas foram analisadas e mediante consenso, foram acatadas, visando conferir maior fidedignidade ao cenário.

\subsection{Simulação}

O município possui 32 unidades de Estratégia de Saúde da Família (ESF) e 11 Unidades de Saúde Tradicional na rede pública. Previamente à simulação, para que a cena se tornasse ainda mais real, foram realizadas visitas em cinco UBS indicadas pela coordenação da Atenção Primária em Saúde do município. Tais visitas foram realizadas pelo ator/pesquisador e este participou ativamente da vivência das salas de vacinação visitadas, observando a rotina das unidades e o perfil de atendimento à população a fim de trazer mais fidedignidade à cena.

A simulação foi conduzida por 5 pesquisadores e 5 estudantes de pós-graduação. Entre eles, uma pesquisadora especialista na área de vacinas e um facilitador, com experiência na área de simulação realística. Assim, para cada evento da simulação, foram elaboradas ações específicas que se esperam que um profissional realize no ato da vacinação. Essas ações se basearam em dimensões inerentes ao processo de registro, a saber: 1) dimensão do registro do vacinado e 2) dimensão movimento dos imunobiológicos (Silva, Coelho, Cavalcante, Oliveira, \& Guimarães, 2018).

No Brasil, o Programa Nacional de Imunizações (PNI) recomenda que as atividades em salas de vacinação sejam realizadas por uma equipe de enfermagem capacitada (Ministério da Saúde, 2017). Desta forma, a simulação foi executada com profissionais atuantes em salas de vacinação das UBS, tendo sido convidados enfermeiros e técnicos de enfermagem a responderem o Checklist para Avaliação do desempenho do Sistema de Informação de Imunização (CASII) (Ferreira et al., 2021), checklist validado no contexto brasileiro, para analisar o desempenho de suas atividades referentes a gestão e registro de imunização.

Entre os que responderam ao questionário $(\mathrm{n}=103)$, foram selecionados aqueles que obtiveram grau de desempenho menor que 50\% ( $n=22)$, considerado não adequado e, portanto, a recomendação foi a capacitação. Um total de 12 participantes, sendo 6 enfermeiros e 6 técnicos de enfermagem concordaram em participar da simulação, sendo esclarecidos e dando anuência mediante assinatura do termo de consentimento livre e esclarecido.

Salienta-se que a simulação foi elaborada a partir de padrões para atender aos seguintes quesitos de qualidade, tomando-se como princípio o referencial metodológico proposto na literatura (Jeffries, 2005; Jeffries \& Rogers, 2012): 1) objetivos da simulação; 2) fidelidade; 3) resolução de problemas e 4) debriefing, conforme descrito a seguir:

Para atender ao "objetivo da simulação" foi estabelecido o seguinte objetivo ao profissional que entrasse em cena: "Você deverá realizar o atendimento de enfermagem ao indivíduo vacinado e operar o SIS".

Para atender ao quesito "fidelidade", realizou-se a simulação em uma sala de vacinação real para que os ruídos externos à UBS pudessem mimetizar uma cena real, ou seja, os barulhos de pessoas entrando e saindo da unidade à procura por atendimento, as interrupções na porta de entrada da sala de vacinação e até mesmo a rotina do serviço. Ademais, a sala real contou com todo material necessário para proporcionar um local mais próximo possível da realidade (câmara refrigerada para armazenamento de vacinas, mesa, cadeiras, ampolas, seringas, descarpack, computador, impressos, manuais, entre outros) (Ministério da Saúde, 2017).

Foi eleito um ator/pesquisador levando-se em conta o seu conhecimento frente aos propósitos do estudo e a sua experiência com simulações. Para a elaboração do usuário vacinado (ator/pesquisador) foram observadas características pessoais daqueles usuários que mais procuraram atendimento no período observado nas unidades supracitadas, tal como propõe o 
referencial metodológico (Jeffries, 2005; Jeffries \& Rogers, 2012). Um profissional de saúde atuou juntamente do vacinado simulado (ator/pesquisador) mediante o objetivo da simulação, e os demais, profissionais de saúde não atuantes na cena, foram orientados a observarem as condutas do profissional atuante, tendo tido esta etapa duração de 30 minutos.

Por fim, para atender ao critério "resolução de problemas", foram desenvolvidas as cenas e estipuladas competências e habilidades esperadas do profissional vacinador em cena baseando-se nos domínios propostos: triagem e avaliação da situação vacinal e Registro do vacinado.

\subsection{Debriefing}

Por fim, a terceira e última fase (debriefing) constituiu-se em momento de discussão conjunta coordenada pelo facilitador/pesquisador que se encarregou de realizar um feedback ao profissional/vacinador participante da cena. Esta etapa durou cerca de 60 minutos. O processo de debriefing é considerado o ponto central da aprendizagem baseada em simulação e é definido, na literatura, como o conhecimento adquirido por meio da discussão reflexiva sobre a experiência da simulação, pertinente ao ciclo da aprendizagem experiencial, composto por recordação guiada, reflexão e análise (Klippe, et al., 2020).

O debriefing foi norteado por três perguntas direcionadas ao profissional/vacinador: como foi participar da simulação? Das condutas que realizou, o que você faria de forma semelhante e o que mudaria?; e o que você achou do cenário e do ator?

Todas as etapas da simulação realística (incluindo as falas do debrifing) foram gravadas (em vídeo e áudio) por meio de celular e transcritas na íntegra. Não houve a necessidade de repetição das entrevistas realizadas. A coleta foi encerrada quando os pesquisadores perceberam o atendimento aos elementos propostos no referencial teórico-metodológico (Jeffries, 2005; Jeffries \& Rogers, 2012), o que caracterizou a saturação.

A partir da transcrição do vídeo na íntegra, os elementos relatados foram analisados à luz da técnica de Análise de Conteúdo, modalidade Temático-Categorial (Bardin, 2016). As etapas para a análise compreendem: transcrição; leitura exaustiva do conteúdo para garantir os critérios de validade, como a exaustividade (contemplando todos os aspectos contidos no roteiro); a representatividade (a representação do universo); a homogeneidade (que sejam obedecidos os critérios de escolha em relação a temas, técnicas e interlocutores) e a pertinência (os documentos que forem analisados devem adequar-se aos objetivos do trabalho) (Bardin, 2016). A análise foi procedida por três pesquisadores independentes, que pontuaram suas observações a partir da leitura das transcrições. Em seguida, os resultados foram confrontados e balizados visando um comum acordo e a identificação de categorias por afinidade temática. Destaca-se que as falas foram codificadas de acordo com sua sequência, sendo designadas pela letra "P" (participante) (ex: P1, P2). Os dados transcritos não foram devolvidos aos participantes.

Este estudo foi aprovado pelo Comitê de Ética em Pesquisa da Universidade Federal de São João del-Rei (CAAE n ${ }^{\circ}$ 65656017.6.0000.5545 e parecer nº 3.354.176) e Registro Brasileiro de Ensaios Clínicos sob número de registro (RBR-3cxz3).

\section{Resultados}

A avaliação e validação realizada pelos especialistas levou à elaboração de um cenário para a simulação realística voltado para um atendimento de um profissional de enfermagem em uma sala de vacinação (Figura 1). 
Figura 1. Cenário elaborado para a simulação realística de um atendimento em sala de vacinação. Minas Gerais, Brasil, 2019.

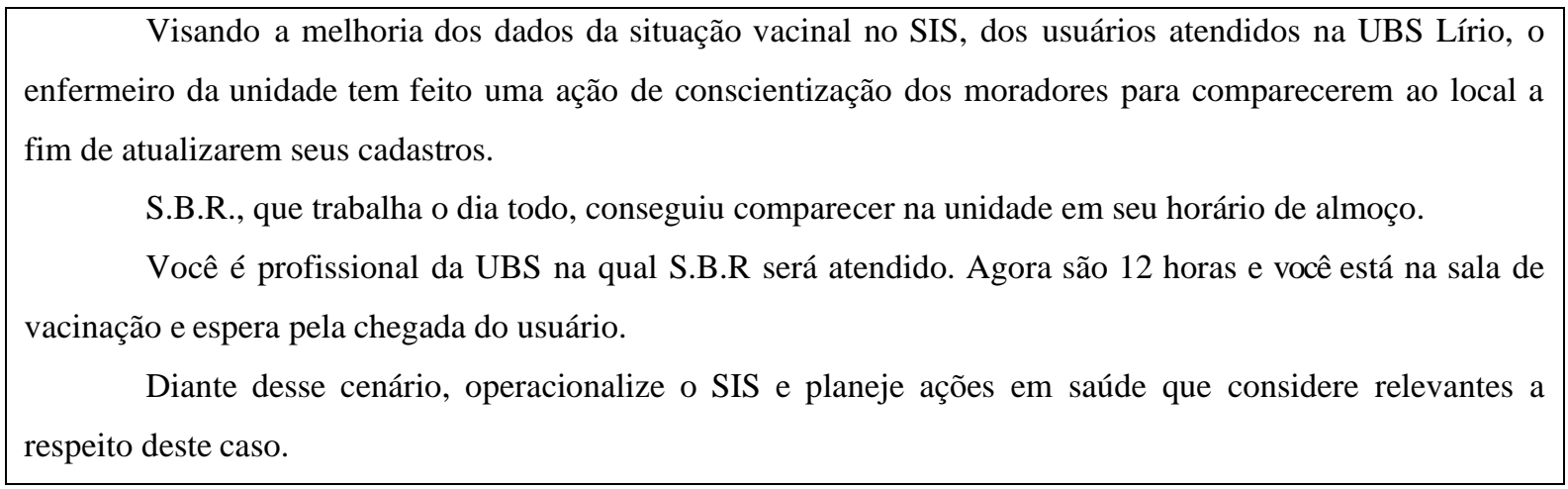

Fonte: Autores (2021).

Este cenário foi avaliado quanto ao aprimoramento de alguns quesitos quanto à sua clareza, objetividade e pertinência. Esta etapa foi fundamental para garantir um produto de qualidade a ser utilizado nesta e em outras capacitações para profissionais atuantes em salas de vacinação.

Quanto à parte escrita do cenário, os especialistas sugeriram algumas alterações: substituição de palavras que pudessem dar mais clareza à cena, tais como "paciente" por "pessoa"; "operacionalize o SIS" por "avalie a situação vacinal do indivíduo e realize as ações de imunização, caso necessário"; "diante desse cenário, operacionalize o SIS" por "diante desse cenário, avalie a situação vacinal da pessoa e realize as ações necessárias de imunização”. Os especialistas não apontaram a necessidade de modificação no cenário e nas etapas, por identificarem concordância ao objetivo proposto.

As ações esperadas dos profissionais, presentes no checklist do facilitador, sofreram algumas alterações de conteúdo, sobretudo no que se refere a todos os elementos constituintes dos domínios propostos na literatura (Silva et al., 2018). O cenário, apesar de conter elementos importantes referentes ao registro do vacinado, não contemplou na íntegra todos os quesitos da operacionalização do SIS, uma vez que alguns quesitos abarcam outras complexidades que não puderam ser captadas nesta capacitação. As questões do domínio "movimento dos imunobiológicos" foram excluídas do checklist, uma vez que se referiam a ações de tomada de decisão de caráter mais complexo, desenvolvidas a longo prazo, não podendo, portanto, serem captadas na simulação ora apresentada. Questões pertencentes aos domínios "triagem e avaliação da situação vacinal" e "registro do vacinado" permaneceram no checklist.

O checklist foi composto das seguintes questões: “As vacinas administradas no usuário são registradas em tempo real no SIS?; "A caderneta de vacinação do vacinado é visualizada para avaliar as doses registradas"?; "É realizado o registro das vacinas administradas anteriormente (registro anterior) no SIS”?; “É selecionada a situação vacinal do usuário (gestante, contato de hanseníase) no momento do registro"?; "É conferido o lote da vacina cadastrado que será administrada no vacinado quando há mais de um lote cadastrado no SIS"?

No conjunto dos registros dos vídeos, identificaram-se 13 unidades de significado e 13 códigos que foram agrupados em 2 categorias específicas, as quais emergiram por afinidade temática, mediante balizamento das análises obtidas de cada um dos pesqusiadores, a saber: categoria 1: Fidelidade e metodologia inovadora em educação permanente e categoria 2: Resolução de problemas.

\subsection{Fidelidade e metodologia inovadora em educação permanente}

Essa categoria abordou questões que permearam sobre o quanto a simulação realizada se aproximou da rotina das unidades nas quais os profissionais trabalham. Percebeu-se ainda a importância de se discutir temáticas inerentes à rotina de forma mais próxima da realidade, uma vez que tais práticas podem ser eficazes enquanto ferramentas de educação permanente. 
O uso da simulação parece ser útil para trabalhar a temática com os profissionais de forma mais proveitosa e participativa, como destacado através das falas dos participantes:

Muito próximo da realidade mesmo! Da rotina né? Achei que foi ótimo também! Essa metodologia foi ótima. (P1) Achei muito próximo do que a gente vive. A rotina toda ainda! Achei produtivo, a gente não ficou com sono, a gente aprende mais, a gente participa ativamente. (P2)

Achei que foi muito bem elaborada. Eu nem queria participar no início, pois estou acostumada com palestras e geralmente não gosto...Pensei que fosse isso. Depois que vi que era uma simulação eu pensei não sou atriz, (risos) mas me convenceram a vir. Aí acabou que eu participei né? Foi muito produtivo! (P5)

\subsection{Resolução de problemas}

Nessa categoria foram abordadas falas no que diz respeito aos problemas vivenciados no dia-a-dia nas salas de vacinação que envolvem a tomada de decisões, ou seja, um processo que envolve desde a condução do atendimento e fatores dificultadores na prática até os agentes estressores externos que implicam na assistência. Os profissionais elencaram questões de suas dificuldades ao analisar os cartões de vacinação, manuseio do SIS e os diversos usuários que atendem em seu cotidiano representando alguns fatores estressores externos. E ambos os desafios enfrentados foram elencados na simulação.

O tipo de paciente, o cartão que a gente pega, a situação de tá no SIS. Da forma como vimos na simulação ajuda muito na tomada de decisão porque é exatamente aquilo alí que foi mostrado. (P7)

Os usuários lá fora reclamando da falta de tempo e demora de atendimento... A questão do cartão que não tá no sistema... A paciente estressada... achei que eu estivesse mesmo onde trabalho e depois dessa simulação me sinto mais segura caso apareça este caso na unidade. (P8)

\section{Discussão}

A avaliação do cenário pelos especialistas levou ao aprimoramento do cenário quanto à clareza, objetividade e pertinência, etapas fundamentais na efetividade de capacitações. De acordo com o modelo proposto no estudo (Jeffries, 2005; Jeffries \& Rogers, 2012) percebeu-se que os especialistas optaram pela substituição de algumas terminologias para facilitar a compreensão do cenário. Assim, foi utilizada uma linguagem padrão para descrição das informações que podem ajudar pesquisadores a desenvolver cenários e instrumentos psicométricos para replicação de estudos.

Após passar pelo crivo dos especialistas, a simulação elaborada trouxe um objetivo mais consistente e com maior fidelidade na resolução de problemas, com destaque à atuação do facilitador no processo de ensino-aprendizagem. Outro estudo aponta sucesso nesta mesma trajetória, indicando que o facilitador deve guiar o caminho a ser percorrido pelos participantes e assim, garantir que todas as habilidades esperadas sejam desenvolvidas, alcançando o objetivo final da cena simulada (Kaneko \& Lopes, 2019).

Foi previamente determinado que a atividade ocorreria entre 15 e 20 minutos. As simulações realísticas precisam imitar a realidade clínica além de serem autênticas. Ademais, devem incluir o maior número de fatores ambientais possíveis, de forma a se aproximar mais da realidade, para assim promover um melhor resultado da aprendizagem (Jeffries, 2005; Jeffries \& Rogers, 2012). Logo, o tempo recomendado para a realização do cenário deve ser equiparado ao tempo real da cena. O tempo previsto mostrou-se suficiente para o desenvolvimento das atividades, tal como ilustrado pelas falas dos participantes.

Outro aspecto importante a ser destacado é com relação aos benefícios da simulação, mais especificamente à segurança do usuário. O modelo de práticas seguras, baseado na segurança do usuário, tem sido associado muitas vezes à 
utilização da simulação realística, apontada como necessária para o preparo do profissional, pois este será treinado para agir, com segurança, em momentos de tensão, nos quais é necessária a aplicação do julgamento clínico e pensamento crítico para que se tenha sucesso em seu raciocínio clínico tanto diagnóstico, como terapêutico (Kaneko \& Lopes, 2019; Shoji et al., 2021). No caso das salas de vacinação é importante que o profissional de enfermagem tenha conhecimento e prática de suas habilidades clínicas e de gestão, sobretudo na propagação de informações e práticas seguras quanto ao manuseio das vacinas (Ministério da Saúde, 2017; Amaral et al., 2020).

No que se refere ao referencial metodológico proposto (Jeffries, 2005; Jeffries \& Rogers, 2012), os resultados sugerem que o cenário obteve um objetivo claro e coerente aos propósitos do estudo. Por isso, torna-se fundamental a construção de cenários clínicos cujos objetivos estejam claros e que permitam ao aluno e ao profissional de enfermagem se aproximar ao máximo da realidade clínica, uma vez que este será capaz de melhorar sua satisfação ao participar da vivência prática e que permitam também uma avaliação contínua de todo o processo envolvido (Cogo et al., 2019; Shoji et al., 2021).

A proximidade do cenário construído pôde ser constatada também pelas falas dos participantes, os quais relataram sensações, de maneira geral, positivas frente a esta técnica. $\mathrm{O}$ uso desta metodologia ativa trouxe experiências bem próximas da realidade dos serviços de saúde aos quais os sujeitos estão envolvidos.

Quanto à fidelidade, percebe-se que a simulação consegue essa aproximação com o cotidiano vivenciado pelos profissionais. Ademais, outro estudo aponta que cenários de simulação devem oferecer experiências cognitivas, psicomotoras e afetivas, contribuindo para a transferência de conhecimento da sala de aula para os ambientes clínicos, então a duração do cenário deve corroborar com a ideia da realidade, pois após essa experiência, os docentes devem reforçar os acertos nos procedimentos, corrigir os erros e explicar os pontos nos quais há necessidade de aprimoramento dos alunos (Souza et al., 2018). Neste estudo percebeu-se que estes elementos foram contemplados de forma efetiva, diante das falas dos participantes.

$\mathrm{Na}$ etapa final da experiência clínica simulada (debriefing) os participantes também apontaram elementos que evidenciaram sua satisfação de aprendizagem diante do cenário clínico apresentado perante o seu realismo. Embora a simulação não possa substituir a experiência clínica, pode, contudo, oferecer oportunidades para o estudante aprender em cenários reais (Almeida et al., 2016; Shoji et al., 2021), a exemplo das salas de vacinação.

Acredita-se ainda que a riqueza das discussões apresentadas pelos participantes tenha sido em parte pelo fato de o debriefing ter sido realizado sequencialmente à simulação. Recomenda-se que o debriefing seja realizado dessa forma para que os pensamentos e sentimentos vivenciados durante a atividade não sejam esquecidos ou fiquem distorcidos, devido ao passar do tempo, e deve incluir os pesquisadores e profissionais que participaram do processo (Almeida et al., 2016). No presente estudo, a questão que versa sobre o uso de um ambiente idêntico ao que a simulação ocorreu foi considerada positiva.

Diante disso, infere-se que o uso de simulações realísticas nas capacitações em salas de vacinação pode favorecer o aperfeiçoamento de técnicas dos profissionais de forma ética e segura para o usuário. Ademais, propicia um método inovador de educação a partir do desafio enfrentado na assistência e desenvolve aos participantes a reflexão de práticas e condutas seguras (Yamane, Machado, Osternack, \& Mello, 2019). Essa metodologia se faz importante não somente para os profissionais atuantes nos serviços de saúde, mas também aos estudantes de graduação enquanto futuros profissionais nesses serviços (Mesquita, Santana, \& Magro, 2019; Shoji et al., 2021).

Quando o profissional refere que a metodologia se aproxima da realidade em que trabalha, esta se apresenta como útil para a identificação dos problemas e a sua resolução, podemos perceber o quanto a simulação se apresenta efetiva quanto aos desafios vivenciados no cotidiano, e desenvolve uma autoconfiança a partir da experiência e discussão vividas. Consequentemente, quando a cena se torna mais fidedigna a autoconfiança na tomada de decisões também aumenta, sendo esta, essencial para a realização das técnicas. Para a resolução dos problemas diários são necessários experiências, aprendizagem efetiva e reflexões de suas condutas. Diante disso, o uso da simulação realística com os profissionais estimula o 
desenvolvimento de habilidades cognitivas, psicomotoras e de atitudes, incentivados pelo conhecimento teórico em prática (Mesquista et al., 2019).

Ao analisar estes resultados sob à luz do referencial teórico adotado, destacam-se domínios importantes amplamente desenvolvidos e que obtiveram resultados positivos, sendo os domínios afetivos e psicomotores, uma vez que os participantes se comoveram com a cena e conseguiram desenvolver de forma adequada as habilidades técnicas que são inerentes à vacinação e ao manuseio do SIS. Portanto, em visão dos princípios da Taxonomia de Bloom, foi possível observar elementos positivos, tal como também evidenciado por outros estudos (Negri, Pereira Jr, Cotta Filho, Franzon, \& Mazzo, 2019; Gonçalves, Bórnea, \& Padovani, 2020; Mazzo et al., 2018).

Durante o desenvolvimento da simulação, os objetivos de ensino-aprendizagem foram definidos de acordo com os resultados almejados. A utilização da Taxonomia de Bloom, conforme sua classificação hierárquica dos objetivos de aprendizagem, também possibilitou procurar meios de estimular o raciocínio e abstrações de alto nível, sem distanciar-se do desempenho que os educadores gostariam que os alunos manifestassem antes de conhecerem determinados assuntos (Hanshaw $\&$ Dickerson, 2020).

Acredita-se que este estudo possui potencial inovador e seja precursor na melhoria da tomada de decisões frente aos desafios impostos pela rotina de trabalho nas mais diversas áreas de atuação em saúde, as quais, muitas vezes, são complexas e exigem um engajamento dos profissionais de enfermagem para trabalharem em equipe e alcançar resultados promissores.

Além disso, esta metodologia pode facilitar o manuseio de tecnologias leves, representadas pelos SIS os quais, continuamente, têm sido implementadas pelos serviços de saúde (Cavalcante, Kerr-Pinheiro, Guimarães, \& Miranda, 2015). Atrelado a isso, é comum aos profissionais de enfermagem apresentarem certa resistência quanto ao uso de SIS. Desta maneira, acredita-se que as simulações, ao se aproximarem da realidade dos serviços destes profissionais, consigam ter potencial sensibilizador para mudança de comportamento e, consequentemente, melhoria na assistência e registro das informações de forma correta.

Portanto, a utilização de cenários previamente elaborados e validados pode colaborar efetivamente com o êxito na utilização da simulação enquanto estratégia de ensino. Assim, disponibilização de cenários validados em estudos futuros e a capacitação de mais profissionais para atuar com essa estratégia de ensino, podem contribuir para garantir a simulação com qualidade.

Uma das limitações deste estudo compete ao fato de que a elaboração e o desenvolvimento do cenário requerem tempo e novas habilidades, o que pode ser caracterizado como uma dificuldade da simulação, visto que muitos profissionais dos serviços de saúde, por não conhecerem a técnica, a princípio desacreditaram na realização das atividades. Outra limitação se refere ao fato de que, como esta metodologia consiste em uma estratégia de ensino inovadora que segue certo rigor nas etapas na execução, nem sempre os serviços de saúde contarão com profissionais capacitados para replicá-la da forma como foi procedido neste estudo.

\section{Conclusão}

Este estudo objetivou descrever a elaboração de um cenário de simulação realística para capacitação em salas de vacinação, cujo objetivo foi que o profissional de enfermagem operacionalizasse o SIS e planejasse ações em saúde que considerasse relevantes a respeito do caso apresentado.

Destaca-se a relevância deste estudo pela apropriação de uma técnica inovadora e que, apesar de possuir um método sistematizado, facilmente pode ser replicado a profissionais de saúde atuantes em diversos setores, a exemplo das UBS, ambulatórios e hospitais, respectivamente. Entre as vantagens destaca-se o baixo custo para execução da técnica, podendo ser utilizados os materiais e o espaço físicos da própria instituição. Além disso, a técnica não requer um preparo minucioso do 
ator, tal como proposto neste estudo, uma vez que as simulações podem se replicar a realidade com um nível de complexidade reduzido, a depender dos objetivos de quem propõe a capacitação.

Portanto, percebe-se ainda mais a necessidade de sensibilizar gestores, profissionais e outros indivíduos que estejam direto ou indiretamente envolvidos com as rotinas dos serviços de saúde, a fim de que esta técnica se torne mais popularizada e próxima das realidades em questão. De forma geral, esta realidade requer uma mudança cultural na percepção da rotina dos serviços das UBS, uma vez que em muitas delas os profissionais se destinam exclusivamente à realização de procedimentos habituais e atividades de gestão, não priorizando o uso de capacitações aprimoradas.

\section{Agradecimentos}

Fundação de Amparo à Pesquisa de Minas Gerais e Ministério da Saúde - Programa de Pesquisa para o SUS - PPSUS (APQ-01505-17); Coordenação de Aperfeiçoamento de Pessoal de Nível Superior (Bolsa CAPES PNPD) (código 001).

\section{Referências}

Almeida, R. G. S., Mazzo, A., Martins, J. C. A., Coutinho, V. R. D., Jorge, B. M., \& Mendes, I. A. C. (2016). Validation to Portuguese of the Debriefing Experience Scale. Revista Brasileira de Enfermagem, 69(4):658-64.

Alvarez, L. N. R., \& Moya, J. L. M. (2017). El prácticum: eje formador de la práctica reflexiva en enfermería. Hácia lá Promoción de la Salud, 22 (1):70-83.

Amaral, G. G., Oliveira, V. C., Guimarães, E. A. A., Reis, I. A., Viegas, S. M. F., Pinto, I. C., \& Oliveira, M. M. (2020). Evaluation of the psychometric properties of the Immunobiological Agent Conservation Assessment Scale. Journal of Nursing Measurement, 28(3):1-18.

Bardin, L. (2016). Análise de conteúdo. Edições 70.

Braz, R. M., Domingues, C. M. A. S., Teixeira, A. M. S, \& Luna, E. J. A. (2016). Classification of transmission risk of vaccine-preventable diseases based on vaccination indicators in Brazilian municipalities. Epidemiologia e Serviços de Saúde, 25(4):745-54.

Cavalcante, R.B., Kerr-Pinheiro, M.M., Guimarães, E.A.A., \& Miranda, R.M. (2015). Panorama de definição e implementação da Política Nacional de Informação e Informática em Saúde. Cadernos de Saúde Pública, 31(5):960-70.

Cavalcante, R. B., Vasconcelos, D. D., Gontijo, T. L., Guimarães, E. A. A., Machado, R. M., \& Oliveira, V. C. (2018). Computerization of primary health care information systems: advances and challenges. Cogitare Enfermagem, 23(3):e54297.

Cogo, A. L. P., Lopes, E. F. S., Perdomini, F. R. I., Flores, G. E., \& Santos, M. R. R. (2019). Building and developing realistic simulation scenarios on safe drug administration. Revista Gaúcha de Enfermagem, 40(spe): e20180175.

Community Preventive Services Task Force. (2015). Recommendation for use of immunization information systems to increase vaccination rates. Journal of Public Health Management \& Practice, 21(3):249-52.

Ferreira, A. P., Silva, B. S., Pereira, M. A. D., Oliveira, V. C., Quites, H. F. O., Amaral, G. G., \& Guimarães, E. A. A. (2021). Checklist para avaliação do desempenho do Sistema de Informação de Imunização: desenvolvimento e validação. Revista Cubana de Información en Ciencias de la Salud, 32 (1): e1688.

Gonçalves, A., Bórnea, E. R., \& Padovani, C. R. (2020). Explorando a formação médica em hanseníase pela aplicação da taxonomia de Bloom. Revista de Graduação USP, 4(1):19-27.

Hanshaw, S. L., \& Dickerson, S. S. (2020). High fidelity simulation evaluation studies in nursing education: A review of the literature. Nurse Education in Practice, 46:102818.

Jeffries, P. R. (2005). A framework for designing, implementing, and evaluating simulations used as teaching strategies in nursing. Nursing Education Perspective, 26(2):96-103.

Jeffries, P. R., \& Rizzolo, M. A. (2006). Designing and implementing models for the innovative use of simulation to teach nursing care of ill adults and children: a national, multi-site, multi-method study. National League for Nursing.

Jeffries, P. R., \& Rogers, K. J . (2012). Theoretical framework for simulation design. In: Jeffries PR. Simulation in Nursing Education: From Conceptualization to Evaluation. New York: National League for Nursing.

Kaneko, R. M. U., \& Lopes, M. H. B. M. (2019). Realistic health care simulation scenario: what is relevant for its design? Revista da Escola de Enfermagem da USP, 53:e03453.

Klippe, C. S. C., Nieto, E. C. B., Santos, H. A. S., Emmerick, L. G., Costa, L. C. R., \& Silva R. C. L. (2020). Contribution of debriefing in simulation-based on simulation. Revista de Enfermagem da UFPE, 14: e241872.

Mazzo, A., Miranda, F. B. G., Meska, M. H. G., Bianchini, A., Bernardes, R. M, \& Pereira Jr, G. A. (2018). Teaching of pressure injury prevention and treatment using simulation. Escola Anna Nery, 22(1): e20170182. 
Mesquita, H. C. T., Santana, B. S., \& Magro, M. C. S. (2019). Effect of realistic simulation combined to theory on self-confidence and satisfaction of nursing professionals. Escola Anna Nery, 23(1):e20180270.

Ministério da Saúde. (2017). Secretaria de Vigilância em Saúde. Departamento de Vigilância Epidemiológica. Manual de rede de frio. (5a ed.), Brasília.

Negri, E. C., Pereira Jr, G. A., Cotta Filho, C. K., Franzon, J. C., \& Mazzo, A. (2019). Construction and validation of simulated scenario for nursing care to colostomy patients. Texto \& Contexto Enfermagem, 28:e20180199.

Oliveira, S. N., Massaroli, A., Martini, J. G., \& Rodrigues, J. (2018). From theory to practice, operating the clinical simulation in Nursing teaching. Revista Brasileira de Enfermagem;71(suppl 4):1791-8.

Pereira, A. S., Shitsuka, D. M., Parreira, F. J., \& Shitsuka, R. (2018). Metodologia da pesquisa científica. UFSM.

Pinheiro, A. L. S., Andrade, K. T. S., Silva, D. O., Zacharias, F. C. M., Gomide, M. F. S., \& Pinto, I. C. (2016). Health management: the use of information systems and knowledge sharing for the decision-making process. Texto \& Contexto Enfermagem, 25(3):1-9.

Santos, A. F., Sobrinho, D. F., Araújo, L. L., Procópio, C. S. D., Lopes, E. A. S., Lima, A. M. L. D., Reis, C. M. R., Abreu, D. M. X., Jorge, A. O., \& MattaMachado, A. T. (2017). Incorporação de Tecnologias de Informação e Comunicação e qualidade na atenção básica em saúde no Brasil. Cadernos de Saúde Pública, 33(5):e00172815.

Sato, A. P. S. (2015). National Immunization Program: Computerized System as a tool for new challenges. Revista de Saúde Pública, 49(39):1-5.

Scarparo, A. F., Laus, A. M., Azevedo, A. L. C. S., Freitas, M. R. I., Gabriel, C. S., \& Chaves, L. D. P. (2012). [Reflections on the use of delphi technique in research in nursing]. Revista da Rede de Enfermagem do Nordeste, 13(1):242-51.

Shoji, S., Avena, D. A., Carvalho, E. C., Soares, S. S. S., Varella, T. C. M. y M., Farias, S. N. P., Andrade, K. B. S., Pereira, S. R. M., \& Souza, N. V. D. O. (2021). A formação de egressos de Enfermagem e seus estranhamentos no mundo do trabalho em saúde. Research, Society and Development, 10(1):e18110111558.

Silva, B. S., Coelho, H. V., Cavalcante, R. B., Oliveira, V. C., \& Guimarães, E. A. A. (2018). Evaluation study of the National Immunization Program Information System. Revista Brasileira de Enfermagem, 71(supll 1):615-24.

Souza, E. F. D., Silva, A. G., \& Silva, A. I. L. F. (2018). Active methodologies for graduation in nursing: focus on the health care of older adults. Revista Brasileira de Enfermagem, 71 (suppl 2):920-4.

Troncon, L. E. A., \& Maffei, C. M. L. (2007). A incorporação de recursos de simulação no curso de graduação em Medicina da Faculdade de Medicina de Ribeirão Preto - USP. Medicina (Ribeirão Preto, Online), 40(2):153-61.

Yamane, M. T., Machado, V. K., Osternack, K. T., \& Mello, R. G. (2019). Simulação realística como ferramenta de ensino na saúde: uma revisão integrativa. Revista Espaço para a Saúde, 20(1):87-107. 